\title{
Height Restoration after Balloon Kyphoplasty in Rheumatoid Patients with Osteoporotic Vertebral Compression Fracture
}

\author{
Seung-Pyo Suh ${ }^{1}$, Chul-Woong Kim ${ }^{2}$, Young-Hoon Jo ${ }^{2}$, Chang-Nam Kang ${ }^{2}$ \\ ${ }^{1}$ Department of Orthopedic Surgery, Sung-Ae Hospital, Seoul, Korea \\ ${ }^{2}$ Department of Orthopedic Surgery, Hanyang University College of Medicine, Seoul, Korea
}

\begin{abstract}
Study Design: Retrospective study.
Purpose: This study was conducted to compare vertebral body height restoration rate in rheumatoid arthritis (RA) patients who had undergone percutaneous balloon kyphoplasty (KP) with that of control group who had matched age, sex, body mass index, and bone mineral density.

Overview of Literature: There is no report on result of KP in RA patients.

Methods: Postoperative height restoration rate of RA group consisting of 15 patients (18 vertebral bodies) who had undergone KP due to osteoporotic vertebral compression fracture with a 30\% or higher vertebral compression rate between May 2005 and January 2013 were compared to control group consisting of 38 patients (39 vertebral bodies) who had matched age, sex, body mass index, and bone mineral density.

Results: No statically significant difference in age $(p=0.846)$, sex $(p=0.366)$, body mass index $(p=0.826)$, bone mineral density $(p=0.349)$, time to surgery $(p=0.528)$, polymethylmethacrylate injection time $(p=0.298)$, or amount $(p=0.830)$ was found between the RA group and the control group. However, preoperative compression rate in the RA group was significantly $(p=0.025)$ higher compared to that in the control group. In addition, postoperative height restoration rate showed significant correlation with the RA group $(p=0.008)$. Although higher incidence of recollapse occurred in the RA group compared to that in the control group, the difference was not statistically significant $(p=0.305)$.

Conclusions: Compared to the control group, RA patients showed higher compression rate and higher vertebral restoration rate after $\mathrm{KP}$, indirectly indicating weaker bone quality in patients with RA. Higher incidence of recollapse occurred in the RA group compared to that in the control group, although it was not statistically significant.
\end{abstract}

Keywords: Rheumatoid arthritis; Thoracolumbar spine; Osteoporotic vertebral compression fracture; Balloon kyphoplasty; Height restoration rate

\section{Introduction}

Rheumatoid arthritis (RA) is widely known to be one of the most frequent inflammatory arthritis conditions, affecting $0.5 \%-1 \%$ of the entire population. Osteoporosis and osteoporotic vertebral compression fractures are

Received Oct 15, 2014; Revised Dec 30, 2014; Accepted Dec 30, 2014

Corresponding author: Chang-Nam Kang

Department of Orthopaedic Surgery, Hanyang University College of Medicine,

222 Wangsimni-ro, Seongdong-gu, Seoul 133-791, Korea

Tel: +82-02-2290-8485, Fax: +82-02-2299-3774, E-mail: cnkang65@hanyang.ac.kr 
well-known complications of RA [1]. The prevalence of osteoporosis in RA patients has been reported to be at least twice as high as that in the non-RA population [2], thus increasing the risk of osteoporotic vertebral compression fracture by two to three times and the risk of spine fracture by 6.2 times [3,4]. In addition, RA could increase the risk of death from osteoporotic vertebral compression fracture and incidence of new osteoporotic vertebral compression fractures or other fractures $[5,6]$. Therefore, it is critical to manage pain and prevent the progress of the disease to reduce serious complications through early treatment of osteoporotic vertebral compression fractures.

Among treatment modalities of osteoporotic vertebral compression fractures, percutaneous balloon kyphoplasty (KP) has been found to enable early walking by restoring the height of collapsed vertebra, thus reducing pain and decreasing complications as well as improving the quality of life of patients. Although studies on the effect and prognosis of percutaneous balloon KP have been well reported, there is no report on the result of percutaneous balloon KP on RA patients whose risk of osteoporotic vertebral compression fracture is twice as high compared to the non-RA population. Therefore, the objective of this study was to determine vertebral height restoration rate in RA patients compared to that in control (non-RA) group after percutaneous balloon KP. Another objective was to identify factors that could affect height restoration rate after percutaneous balloon KP.

\section{Materials and Methods}

There was no research-related contact with patients. Therefore, our Institutional Review Board (Hanyang University Research Ethics Committee) waived the informed consent and ethical approval. All activity was conducted in accordance with the Declaration of Helsinki and institutional standards.

The RA group consisted of 15 patients (18 cases) who had undergone percutaneous balloon KP due to osteoporotic vertebral compression fracture with a $30 \%$ or higher vertebral compression rate between May 2005 and January 2013. The control group consisted of 38 patients (39 cases) with matched age, sex, body mass index, and bone mineral density. Patients who showed a compression of the vertebral body on the anteroposterior and true lateral radiographs of lumbosacral or thoracolumbar in supine position upon hospitalization and tenderness on the corresponding site during physical examination were diagnosed with osteoporotic vertebral compression fracture. A recent fracture was confirmed by noting an increased signal density in T2 weighted images of magnetic resonance imaging scans (Fig. 1). Those who had a history of major trauma or were suspected of infection or malignant tumor were excluded. Patients who satisfied the criteria of the American College of Rheumatology and were followed up by the Rheumatology Department of Hanyang University Hospital were included in the RA group. Their sex, age at the time of surgery, body mass index, time to surgery, bone mineral density, polymethylmethacrylate (PMMA) injection time, and PMMA injection amount were recorded or determined. Radiological measurements were performed twice with two-week intervals by three different personnel (one spine fellow, one clinical professor, and one orthopedic resident). Preoperative compression rate, postoperative height restoration, and recollapse of cemented vertebra were measured with PACS $\pi$ view star (Infinitt, Seoul, Korea) digital measurement application. The means of independent measurement values were set as representative values.

Preoperative compression rate was calculated as percentile of the height of the compressed anterior or middle vertebral body against the mean height of the anterior or middle vertebral bodies of adjacent top and bottom vertebral bodies in lateral radiographs taken in supine position
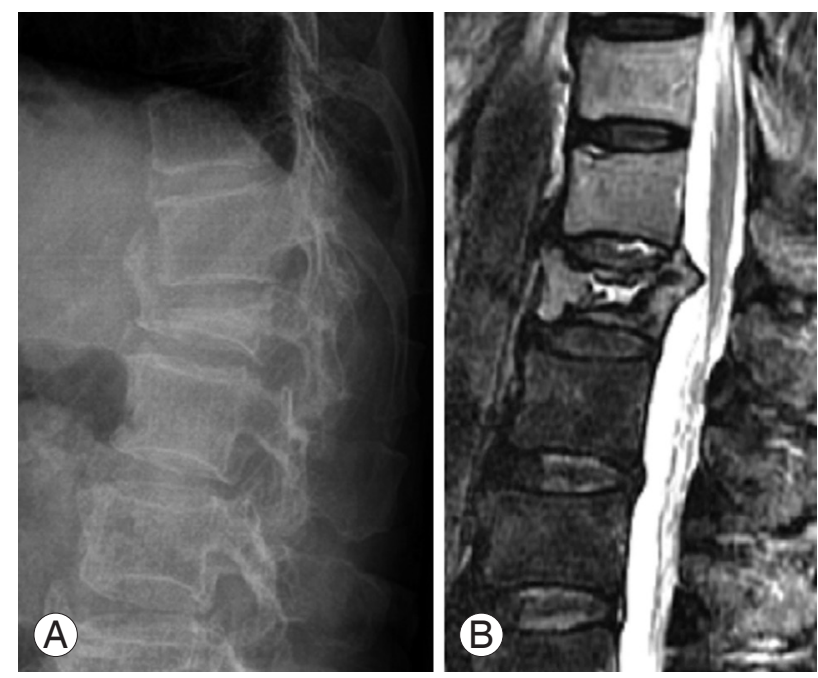

Fig. 1. (A) Lateral radiograph of the thoracolumbar spine showing vertebral compression fracture. (B) T2-weighted magnetic resonance imaging sagittal scan showing increased signal density of the same vertebral body. 
(Fig. 2). Postoperative restoration rate was calculated using a method described by McKiernan et al. [7] based on the values of the anterior or intermediate vertebral bodies in the standing lateral radiograph determined in the same manner as that prior to surgery (Fig. 2). The recollapse of cemented vertebra was diagnosed as recurrent back pain. It was postoperatively correlated with height reduction of cemented vertebra and osteolysis around the cement on serial standing lateral radiograph. Bone mineral density was measured with dual energy X-ray absorptiometry.

The surgery was conducted by one operator (C.N.K.) for all cases that were at least three weeks since the injury date with at least $30 \%$ compression rate. The surgery was conducted within 3 weeks for 15 cases (11\%). In every case, morbid vertebral bodies were checked with C-arm fluoroscopy in a prone position. The bilateral pedicle approach was used with local anesthesia. An inducer was inserted along the spine pedicle at $1 \mathrm{~cm}$ outside the pedicle and positioned slightly below the anterior center of the vertebral body. A balloon tamp was inserted and inflated at a pressure not greater than $300 \mathrm{~mm} \mathrm{Hg}$ to restore the compressed vertebral body. After the removal of the balloon, PMMA was injected through two feeders. The feeders were retracted when the leakage of PMMA was observed and the injection was completed. The injection amount of PMMA was determined by adding up the amounts of cement injected to both sides. PMMA injection time was determined as the duration from the
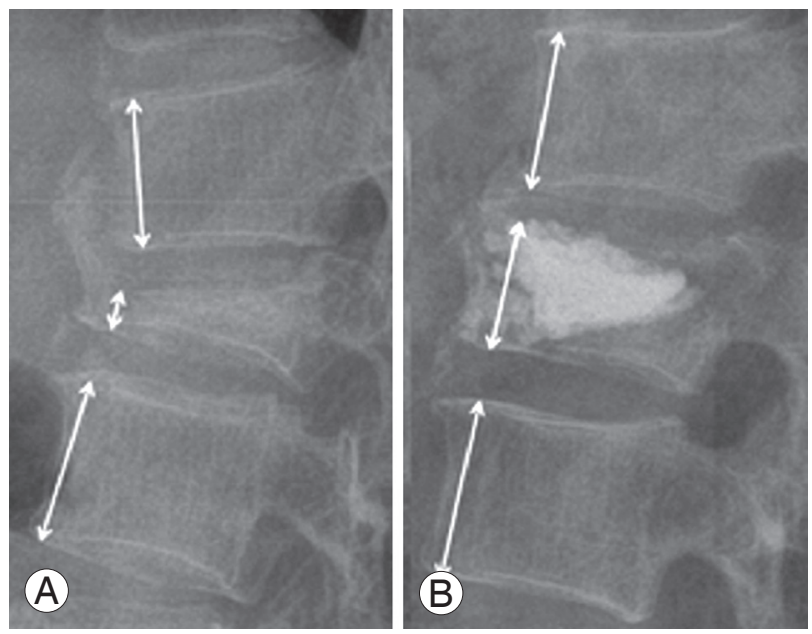

Fig. 2. (A) The preoperative compression rate was calculated as the percentile of the height of the compressed anterior vertebral body against the mean height of adjacent top and bottom vertebral bodies in the lateral radiographs. (B) The postoperative restoration rate was determined in the same manner as that prior to surgery. polymerization of PMMA until the commencement of injection. Patients started to walk from the day of surgery. They were followed up as outpatients for an average of 25.2 months (range, 3-36 months) after the surgery.

For statistical analysis, independent $t$-test was used to compare age, sex, body mass index, time to surgery, bone mineral density, polymethylmethacrylate injection amount and time, and the mean of the preoperative compression rate. Fisher exact test was used to compare the rate of recollapse between the two groups. Postoperative height restoration rate was analyzed with a simple regression analysis. Intra- and interobserver agreements were verified with Cronbach's alpha factor.

\section{Results}

The interobserver reliability of preoperative compression rate and postoperative restoration rate had Cronbach's alpha value of $0.919-0.950$. The intraobserver reliability had Cronbach's alpha value of 0.891-0.940. Therefore, there were excellent intra- and interobserver agreements.

The average age of the 15 patients ( 3 males and 12 females) in the RA group with 18 cases was 70.1 years (range, 58-75 years). The average age of the 38 patients (5 males and 33 females) in the control group with 39 cases was 70.4 years (range, 58-75 years). Sex or age of the two groups was not significantly $(p=0.366$ and $p=0.846)$ different from each other (Table 1).

Initial fractures in the RA group included 1 case of the 11 th thoracic vertebra, 3 cases of the 12 th thoracic vertebra, 9 cases of the first lumbar vertebra, 2 cases of the second lumbar vertebra, 1 case of the third lumbar vertebra, and 2 cases of the fourth lumbar vertebra. Initial fractures in the control group included 1 case of the ninth thoracic vertebra, 1 case of the 10th thoracic vertebra, 1 case of the 11 th thoracic vertebra, 15 cases of the 12 th thoracic vertebra, 15 cases of the first lumbar vertebra, 4 cases of the second lumbar vertebra, 1 case of the fourth lumbar vertebra, and 1 case of the fifth lumbar vertebra. The 12th thoracic vertebra and the first lumbar vertebra accounted for $75 \%$ in the RA group and $77 \%$ in the control group. Three patients in the RA group and one patient in the control group had two or more osteoporotic vertebral compression fractures.

The average time from injury to surgery was 69.1 days (range, 7-130 days), including most patients who had acute thoracolumbar osteoporotic vertebral compression 
Table 1. Demographic data and clinical features

\begin{tabular}{|c|c|c|c|}
\hline Variable & RA group & Control group & $p$-value \\
\hline Number & 15 Patients (18 cases) & 38 Patients (39 cases) & \\
\hline Age (yr), mean age (range) & $70.16(58-75)$ & $70.41(58-75)$ & 0.846 \\
\hline Sex (male:female) & $3: 12$ & $5: 33$ & 0.366 \\
\hline Body mass index $\left(\mathrm{kg} / \mathrm{m}^{2}\right)$ & 24.3 & 24.1 & 0.826 \\
\hline Duration (day) & 88.6 & 60.1 & 0.528 \\
\hline Bone mineral density $\left(\mathrm{g} / \mathrm{cm}^{2}\right)$ & 0.565 & 0.528 & 0.349 \\
\hline Cement (mL) & 6.66 & 6.76 & 0.830 \\
\hline Cement (time) & 10.45 & 10.86 & 0.298 \\
\hline Leakage (case) & 3 & 7 & 1.000 \\
\hline
\end{tabular}

RA, rheumatoid arthritis.

fractures or chronic thoracolumbar osteoporotic vertebral compression fractures that had elapsed after 2 months or more after acute trauma. The average time from injury to surgery in the RA group and the control group was 88.6 and 60.1 days ( $p=0.528$ ), respectively.

Bone mineral density was measured all 53 patients except 8 who had no records in Hanyang University Hospital because they had been checked in other hospitals before hospitalization in our facility. The standard deviation of bone mineral density of all patients was lower than -2.5 . The mean bone mineral densities of the RA group and the control group were $0.565 \mathrm{~g} / \mathrm{cm}^{2}$ and $0.528 \mathrm{~g} / \mathrm{cm}^{2}$ $(p=0.349)$, respectively.

In average, the injected amount of PMMA in semi-solid condition was $6.7 \mathrm{~mL}$ (range, $4-10 \mathrm{~mL}$ ) per vertebral body. The injected amount of PMMA (RA group, 6.66 $\mathrm{mL}$; control group, $6.76 \mathrm{~mL}$ ) and the injection time of PMMA (RA group, 10.45 minutes; control group, 10.82 minutes) demonstrated no significant difference between the two groups ( $p=0.830$ and $p=0.298$ ). PMMA leakage occurred in 3 cases in the RA group and 7 cases in the control group. There was no significant $(p=1.000)$ difference between the two groups. No case showed neurological complications due to PMMA leakage. The recollapse of cemented vertebra occurred more frequently in the RA group (3 cases, 16.7\%) compared to that in the control group ( 3 cases, $7.7 \%$ ) on postoperative serial plain radiographs. However, the difference between the two groups was not statistically significant $(p=0.305)$. The two groups showed no difference either in body mass index (RA group, $24.3 \mathrm{~kg} / \mathrm{m}^{2}$; control group, $24.1 \mathrm{~kg} / \mathrm{m}^{2}$; $p=0.825)$. The preoperative compression rates in the RA group and the control group were 59.01\% and 51.89\% ( $p=0.025)$ in the control group, respectively. Thus, the RA group showed a significantly higher value than the control group. When factors influencing postoperative height restoration were compared between the two groups through simple regression analysis, the RA group showed more significant correlation $(p=0.008)$ with postoperative height restoration rate than the control group.

\section{Discussion}

It has been reported that bone loss in RA patients is caused by the interaction of numerous factors, such as the activity of disease, age, physical activity, bone mineral density, morbidity, and blood tests [8-13]. Considering that an osteoporotic vertebral compression fracture increases the risk of death by $32 \%$, it is meaningful to analyze the effect of balloon KP in RA patients who are at high risk of osteoporotic vertebral compression fracture [5].

Compared to vertebroplasty, percutaneous balloon KP performed recently for the treatment of osteoporotic vertebral compression fractures offers advantage of correcting kyphotic deformity by restoring vertebral height. Percutaneous balloon KP has been found to reduce complications such as pulmonary embolism from cement leakage [14]. The procedure could be performed relatively simply by injecting PMMA into the vertebral body using local anesthesia. Furthermore, it enables early walking by decreasing pain immediately after surgery. Therefore, it can prevent additional complications due to vertebral 
fractures. Voggenreiter [15] reported that balloon KP could reduce arm movement by restoring the collapsed vertebrae in a vertebral fracture and returning the center of gravity to normal position, therefore preventing any additional fracture of the vertebral bodies. Belkoff et al. [16] reported that it was possible to have additional fracture in 30\% of vertebroplasty and in $97 \%$ of cases for percutaneous balloon KP. However, very few research has studied the factors that could influence the height restoration rate of the balloon KP. Ren et al. [17] analyzed the factors that could influence vertebral reduction by performing balloon KP on single vertebras of 43 cases of osteoporotic vertebral compression fractures. They found that the more serious the preoperative vertebral compression rate was, the less the reduction effect became. However, this study was not conducted among RA patients. The mean preoperative compression rate of the RA group in this study was significantly lower, but the postoperative restoration rate of this group was 2.58 times higher than that of the control group.

In this study, the higher restoration rate of balloon KP in the RA group was significant. This might be associated with long term use of glucocorticoid, a commonly prescribed drug for RA patients. Glucocorticoid plays a key role in the treatment of RA. Long-term use of this can be associated with glucocorticoid-induced osteoporosis (GIOP). GIOP is known to show rapid decrease in bone mineral density in its early stages due to anomalous bone loss. However, bone formation decreases when bone resorption increases, as GIOP affects the differentiation of osteocytes and many steps of calcium metabolism [18]. However, for patients who had vertebral fractures, several studies that reported that there was no significant difference in bone mineral density between a test group who used glucocorticoid and a group that did not $[19,20]$. They explained this with a hypothesis that glucocorticoid interfered with the functions of osteoblast and osteoclast, thereby decreasing bone remodeling and bone mineral density. They also proposed that glucocorticoid might induce the extinction of osteocytes, resulting in the destruction of bone quality $[21,22]$. These factors might also increase the incidence of recollapse of cemented vertebra in the RA group. Heo et al. [23] explained that recollapse could be caused by vertebral factor and filler material factor. They suggested that osteonecrosis was the most important predisposing factor for recollapse. In this study, there was no significant difference in factors related to filler material. Therefore, the vertebral factor might be an important predisposing cause for the higher incidence of recollapse in the RA group. Although MRI findings related to osteonecrosis were not evaluated in this study, it is important to consider the relationship between osteonecrosis and recollapse in future research.

For whatever the reason, compared to the control group, RA patients showed higher compression rate and higher vertebral restoration rate after balloon KP, indirectly indicating weaker bone quality in patients with RA. Therefore, performing balloon KP early in RA patients with osteoporotic vertebral compression fractures might be able to more effectively correct deformations through vertebral reduction and continuous prevention of fractures of adjacent vertebras compared to that in non-RA population.

This study has a number of limitations. Firstly, this was a retrospective study with very small sample size. Secondly, among many factors involved in the occurrence of osteoporotic vertebral compression fracture in RA patients, biochemical indices related to the activity of the disease were not considered. In addition, bone quality could not be evaluated because biochemical bone metabolism markers were not measured in all patients. Thirdly, only those patients who showed $30 \%$ or higher vertebral compression rate were the subjects of this study. This was related to the limitation of the Korean medical insurance system. Lastly, no conclusion about the correlation between the radiological height restoration rate of balloon KP and clinical results was derived. Additional studies on this are necessary in the future.

\section{Conclusions}

Although this study did not reveal factors affecting the body height restoration rate after balloon KP in osteoporotic vertebral compression fractures, RA patients showed higher preoperative compression rate and higher restoration rate of balloon KP than non-RA patients. Higher incidence of recollapse also occurred in the RA group compared to that in the control group, although it was not statistically significant.

\section{Conflict of Interest}

No potential conflict of interest relevant to this article was reported. 


\section{References}

1. Kvien TK, Haugeberg G, Uhlig T, et al. Data driven attempt to create a clinical algorithm for identification of women with rheumatoid arthritis at high risk of osteoporosis. Ann Rheum Dis 2000;59:805-11.

2. Haugeberg G, Uhlig T, Falch JA, Halse JI, Kvien TK. Bone mineral density and frequency of osteoporosis in female patients with rheumatoid arthritis: results from 394 patients in the Oslo County Rheumatoid Arthritis register. Arthritis Rheum 2000;43:522-30.

3. Hooyman JR, Melton LJ 3rd, Nelson AM, O'Fallon WM, Riggs BL. Fractures after rheumatoid arthritis: a population-based study. Arthritis Rheum 1984;27: 1353-61.

4. Peel NF, Moore DJ, Barrington NA, Bax DE, Eastell R. Risk of vertebral fracture and relationship to bone mineral density in steroid treated rheumatoid arthritis. Ann Rheum Dis 1995;54:801-6.

5. Kado DM, Duong T, Stone KL, et al. Incident vertebral fractures and mortality in older women: a prospective study. Osteoporos Int 2003;14:589-94.

6. Lindsay R, Silverman SL, Cooper C, et al. Risk of new vertebral fracture in the year following a fracture. JAMA 2001;285:320-3.

7. McKiernan F, Faciszewski T, Jensen R. Reporting height restoration in vertebral compression fractures. Spine 2003;28:2517-21.

8. Lodder MC, de Jong Z, Kostense PJ, et al. Bone mineral density in patients with rheumatoid arthritis: relation between disease severity and low bone mineral density. Ann Rheum Dis 2004;63:1576-80.

9. Gough AK, Lilley J, Eyre S, Holder RL, Emery P. Generalised bone loss in patients with early rheumatoid arthritis. Lancet 1994;344:23-7.

10. van Staa TP, Leufkens HG, Cooper C. The epidemiology of corticosteroid-induced osteoporosis: a metaanalysis. Osteoporos Int 2002;13:777-87.

11. Sinigaglia L, Nervetti A, Mela Q, et al. A multicenter cross sectional study on bone mineral density in rheumatoid arthritis: Italian Study Group on Bone Mass in Rheumatoid Arthritis. J Rheumatol 2000;27: 2582-9.

12. Sambrook PN, Eisman JA, Champion GD, Yeates MG, Pocock NA, Eberl S. Determinants of axial bone loss in rheumatoid arthritis. Arthritis Rheum 1987;
30:721-8.

13. Sinigaglia L, Varenna M, Girasole G, Bianchi G. Epidemiology of osteoporosis in rheumatic diseases. Rheum Dis Clin North Am 2006;32:631-58.

14. Ma XL, Xing D, Ma JX, Xu WG, Wang J, Chen Y. Balloon kyphoplasty versus percutaneous vertebroplasty in treating osteoporotic vertebral compression fracture: grading the evidence through a systematic review and meta-analysis. Eur Spine J 2012;21:184459.

15. Voggenreiter G. Balloon kyphoplasty is effective in deformity correction of osteoporotic vertebral compression fractures. Spine 2005;30:2806-12.

16. Belkoff SM, Mathis JM, Fenton DC, Scribner RM, Reiley ME, Talmadge K. An ex vivo biomechanical evaluation of an inflatable bone tamp used in the treatment of compression fracture. Spine 2001;26: 151-6.

17. Ren H, Shen Y, Zhang Y. Analysis of related factors on the vertebral height restoration of percutaneous kyphoplasty. Chinese J Spine Spinal Cord 2010;1:15.

18. Jacobs JW, de Nijs RN, Lems WF, et al. Prevention of glucocorticoid induced osteoporosis with alendronate or alfacalcidol: relations of change in bone mineral density, bone markers, and calcium homeostasis. J Rheumatol 2007;34:1051-7.

19. Selby PL, Halsey JP, Adams KR, et al. Corticosteroids do not alter the threshold for vertebral fracture. J Bone Miner Res 2000;15:952-6.

20. Naganathan V, Jones G, Nash P, Nicholson G, Eisman J, Sambrook PN. Vertebral fracture risk with longterm corticosteroid therapy: prevalence and relation to age, bone density, and corticosteroid use. Arch Intern Med 2000;160:2917-22.

21. Manolagas SC. Corticosteroids and fractures: a close encounter of the third cell kind. J Bone Miner Res 2000;15:1001-5.

22. Kumagai S, Kawano S, Atsumi T, et al. Vertebral fracture and bone mineral density in women receiving high dose glucocorticoids for treatment of autoimmune diseases. J Rheumatol 2005;32:863-9.

23. Heo DH, Chin DK, Yoon YS, Kuh SU. Recollapse of previous vertebral compression fracture after percutaneous vertebroplasty. Osteoporos Int 2009;20:47380. 